\title{
8
}
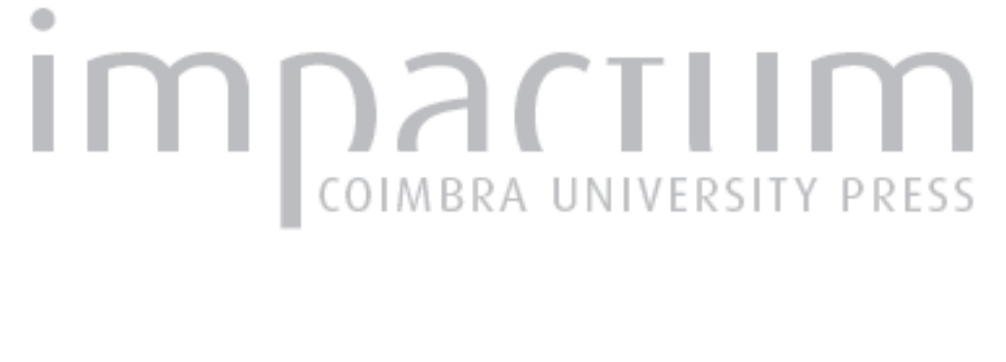

\section{Vulnerabilidade socioambiental de concelhos da Região Centro de Portugal por meio de sistema de informação geográfica}

Autor(es): $\quad$ Freitas, M. Isabel Castreghini; Cunha, Lúcio; Ramos, Anabela
Publicado por: Faculdade de Letras da Universidade de Coimbra, Departamento de Geografia

URL

persistente:

URI:http://hdl.handle.net/10316.2/30262

DOI:

DOI:http://dx.doi.org/10.14195/0871-1623_32_26

\section{Accessed : $\quad$ 26-Apr-2023 08:25:41}

A navegação consulta e descarregamento dos títulos inseridos nas Bibliotecas Digitais UC Digitalis, UC Pombalina e UC Impactum, pressupõem a aceitação plena e sem reservas dos Termos e Condições de Uso destas Bibliotecas Digitais, disponíveis em https://digitalis.uc.pt/pt-pt/termos.

Conforme exposto nos referidos Termos e Condições de Uso, o descarregamento de títulos de acesso restrito requer uma licença válida de autorização devendo o utilizador aceder ao(s) documento(s) a partir de um endereço de IP da instituição detentora da supramencionada licença.

Ao utilizador é apenas permitido o descarregamento para uso pessoal, pelo que o emprego do(s) título(s) descarregado(s) para outro fim, designadamente comercial, carece de autorização do respetivo autor ou editor da obra.

Na medida em que todas as obras da UC Digitalis se encontram protegidas pelo Código do Direito de Autor e Direitos Conexos e demais legislação aplicável, toda a cópia, parcial ou total, deste documento, nos casos em que é legalmente admitida, deverá conter ou fazer-se acompanhar por este aviso.

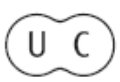




\title{
Vulnerabilidade socioambiental de concelhos da Região Centro de Portugal por meio de sistema de informação geográfica
}

\author{
M. Isabel Castreghini Freitas \\ Departamento de Planejamento Territorial e Geoprocessamento - IGCE. UNESP (Campus de Rio Claro) - Brasil. \\ ifreitas@rc.unesp.br

\section{Lúcio Cunha} \\ Centro de Estudos de Geografia e Ordenamento do Território e Dep. de Geografia. Universidade de Coimbra. \\ luciogeo@ci.uc.pt
}

\section{Anabela Ramos}

Centro de Estudos de Geografia e Ordenamento do Território. Universidade de Coimbra. ana-baia@sapo.pt

\section{Resumo:}

O objetivo principal deste artigo é realizar a análise da vulnerabilidade socioambiental de 17 concelhos que compõem os distritos de Coimbra, Leiria e Santarém (Região Centro de Portugal). A metodologia adotada para a análise da vulnerabilidade baseou-se em Análise Fatorial que agregou variáveis sociais e ambientais estudadas, utilizando o programa de análise estatística SPSS R.18 associado ao programa de Sistema de Informação Geográfica (SIG) ArcGIS 9.3. Os resultados obtidos indicam como principais fatores de vulnerabilidade socioambiental os relacionados com a falta de dinamismo económico e demográfico de parte dos concelhos, principalmente os relacionados com o êxodo rural e a baixa natalidade. 0 modelo gerado mostrou resultados compatíveis com o conhecimento geográfico que detemos para a maioria dos concelhos estudados e espera-se que possa vir a servir de base para propostas de políticas públicas relacionadas com as vulnerabilidades aqui cartografadas.

Palavras-chave: Vulnerabilidade socioambiental. Riscos. Cartografia temática. SIG. Região Centro de Portugal.

\section{Resumé:}

Vulnérabilité sociale et environnementale des communes de la région du Centre du Portugal par l'utilisation du système d'information géographique

L'objectif principal de cette étude est d'effectuer une analyse de la vulnérabilité sociale et environnementale des 17 communes qui comprend les arrondissements municipaux de Coimbra, Leiria et Santarém (Région du Centre du Portugal). La méthodologie adoptée pour l'analyse de vulnérabilité est fondée sur l'analyse factorielle pour l'Analyse en Composantes Principales (ACP), qui a ajutée des aspects sociaux et environnementaux étudiés grâce à l'analyse statistique du programme SPSS R. 18, associé au Système d'Information Géographique (SIG) ArcGIS 9.3. Les résultats indiquent que les facteurs plus importants de vulnérabilité sociale et environnementale sont liés au manque de dynamisme économique de les municipalités et avec le déficit démographique, principalement relative à l'exode rural et le faible taux de natalité. Le modèle généré a montré résultats en accord avec les connaissances géographiques que nous détenons de la plupart des communes étudiées, et il est prévu de fournir une base pour les politiques publiques liées aux vulnérabilités cartographiées ici.

Mots-clés: Vulnérabilité socio-environnementale. Risques. Cartographie thématique. SIG. Région du Centre du Portugal. 


\section{Abstract:}

Social and environmental vulnerability of municipalities of the Central Region of Portugal by using geographic information system

The main aim of this article is to perform an analysis of social and environmental vulnerability of 17 municipalities concerning to the districts of Coimbra, Leiria and Santarém (Central Region of Portugal). The methodology adopted for the vulnerability analysis is based on factor analysis which added social and environmental variables analysed using the statistical program for the social sciences, SPSS R. 18, associated with the Geographic Information System (GIS) ArcGIS 9.3. Using as reference the analysis of cartographic documents, as well as Census of the Statistics Portugal, it was possible to map the main variables for social and environmental risks to which populations are exposed and represent them through thematic maps. The results indicated the main social and environmental vulnerability factors are related to the lack of economic dynamism on the part of Municipalities whose main variables were the access to housing credit and number of enterprises. Also, appeared demographic deficits, mainly related to the ageing population and aspects concerning to quality of life, related with the urban areas, whose most influences in the model were the existence of homes with central heating, rural exodus and the low birth rate. The generated model showed results consistent with the geographic knowledge that we hold for municipalities studied and it is expected that could constitute a basis for future proposals for public policies related to vulnerabilities here mapped.

Keywords: Social and environmental vulnerability. Risks; Thematic mapping. SIG. Central Region of Portugal.

\section{Introdução}

A preocupação com as questões relativas à vulnerabilidade social está na pauta de discussões das grandes organizações mundiais, dentre as quais se destaca a Organização das Nações Unidas (ONU) que, a partir de 2004, vem sistematicamente abrindo agendas visando estudar e traçar diretrizes para fazer face a situações de risco. São considerados riscos naturais os associados ao funcionamento dos sistemas naturais como sismos, cheias e inundações, movimentos em massa, e riscos tecnológicos os que decorrem da atividade humana, como por exemplo potenciais acidentes industriais, acidentes no transporte de substâncias perigosas, entre outros.

De entre tais iniciativas destaca-se a Estratégia Internacional para Redução de Desastres das Nações Unidas (UN-ISDR), que vem realizando reuniões globais a partir de 2007, a cada 2 anos, de acordo com UNISDR (2009). Tal organismo tem a missão de coordenar os parceiros globais e regionais, visando mobilizar compromissos políticos e recursos financeiros para a redução de desastres, desenvolver e manter um sistema de informação de grande porte e promover mecanismos de coordenação multissetorial, através de um sistema de parcerias, com o objetivo de administrar e dar suporte a ações de redução de riscos de desastres (UNISDR, 2011).

Face ao aumento contínuo do risco em áreas urbanas e à deterioração progressiva dos ecossistemas, torna-se imprescindível o traçado de ações integradas, envolvendo governos e parceiros locais, para definir políticas que transformem o conhecimento já consolidado em ações pontuais de projetos-piloto, em ações concretas de larga escala, que se traduzam em benefícios para toda a sociedade.

Neste contexto, surgem as Geotecnologias, em especial os Sistemas de Informação Geográfica, que se apresentam como poderosas ferramentas para a modelação espacial de riscos naturais e tecnológicos e para a cartografia da vulnerabilidade social.

O objetivo principal deste artigo é realizar a análise da vulnerabilidade socioambiental de 17 concelhos dos distritos de Coimbra e Leiria e Santarém (Região Centro de Portugal), selecionados devido à sua localização espacial e à diversidade de características físicas e socioeconómicas que apresentam.

\section{Referenciais teóricos e metodológicos}

Nas últimas décadas, a cartografia de riscos está a avançar, apoiada em ferramentas geotecnológicas cada vez mais sofisticadas, contribuindo assim para estabelecer delimitações espaciais ou zonamento dos níveis de riscos a partir da combinação de mapas de perigosidade, exposição de pessoas e bens e vulnerabilidade.

Considerando-se que o foco deste artigo é a análise da vulnerabilidade socioambiental de 17 Concelhos da Região Centro de Portugal, apresenta-se aqui uma breve revisão de conceitos nas temáticas da vulnerabilidade, cartografia de riscos e das aplicações de Sis- 
tema de Informação Geográfica (SIG), apoiando-se nos referenciais teóricos destas áreas do conhecimento.

$\mathrm{Na}$ busca de definição de Vulnerabilidade merece destaque BLAIIIE et al. (1994) que consideram vulnerabilidade como o conjunto das características de uma pessoa ou grupo, em termos da sua capacidade de antecipar, enfrentar, resistir e se recuperar do impacto de um evento perigoso, seja natural ou tecnológico. Os autores consideram que este conceito implica uma combinação de fatores que determinam o grau em que a vida e o sustento dos indivíduos são colocados em causa por um evento perigoso com origem na natureza ou na sociedade.

Na perspetiva de CUTTER (1996), a vulnerabilidade é um conceito complexo, sob o qual advêm dimensões sociais, económicas, políticas e culturais, cuja definição tem sido abordada, também, sob perspetivas epistemológicas muito diversas da ecologia política, ecologia humana, ciências físicas e análise espacial.

Concorda-se com a postura de BeIER e Dowing (1998) que alertam sobre a medição da vulnerabilidade, que não se deve limitar a dados quantitativos ou fáceis de serem encontrados nos censos, pois corre-se o risco de passarem despercebidos fatores fundamentais da vulnerabilidade que não estão claramente expressos nesses documentos. Nestes casos deve-se levar em conta a complexidade de lidar com tais dados e o desafio constante dos investigadores em garantir a veracidade e pertinência dos parâmetros selecionados para os seus estudos, o que pode ser alcançado por meio da inclusão de dados qualitativos oriundos de consulta aos atores das comunidades envolvidas e de observações de campo.

O estudo de Mendes et al. (2009) propõe um novo índice de vulnerabilidade social aos eventos naturais e tecnológicos perigosos para Portugal que incorpora, além das avaliações padronizadas da exposição e vulnerabilidade biofísica, a resiliência e as capacidades de suporte infraestruturais. Os autores realizaram uma avaliação prévia da vulnerabilidade social para os concelhos de Portugal Continental e uma avaliação à escala da freguesia em sete concelhos da Região Centro, apoiando-se em Sistema de Informação Geográfica para o desenvolvimento de suas análises. 0 estudo pauta-se nos conceitos de criticidade e capacidade de suporte do território. Neste sentido, define criticidade como o conjunto de características individuais e comportamentais que podem contribuir para a ruptura do sistema e conceitua a capacidade de suporte do território como o conjunto de infraestruturas territoriais que permitem à comunidade reagir em caso de desastre.
A metodologia usada para a análise da vulnerabilidade social baseou-se em técnicas estatísticas de análise fatorial que incluiu aspetos sociais e ambientais. Com base nesta estrutura de avaliação foram consideradas duas dimensões: a vulnerabilidade dos indivíduos e comunidades (criticidade) e a vulnerabilidade territorial (capacidade de suporte). Os autores partem do entendimento que, neste tipo de estudo, o lugar e a escala são fundamentais, considerando assim que, de acordo com as características socioeconómicas e territoriais, um mesmo modelo poderá ter bom ou mau desempenho. A experiência de análise à escala nacional (todos os concelhos de Portugal Continental) e municipal (amostra de 7 concelhos na Região Centro, com análises no nível da freguesia) levou os investigadores a concluir que o modelo global se revelou consistente em diferentes escalas, permitindo a definição de estratégias de mitigação do risco e de medidas de proteção civil adaptadas para os locais em estudo (MENDEs et al., 2009: 81).

No âmbito do Brasil destacam-se estudos envolvendo vulnerabilidade social e representação cartográfica, dentre os quais os trabalhos desenvolvidos à escala nacional e municipal que, via de regra, têm adotado como principal base de dados os Censos Demográficos.

O estudo de Garcia e Matos (2007) objetivou avaliar parte das condições de vida das famílias brasileiras e mapear a sua distribuição espacial trabalhando com os microdados do Censo Demográfico de 2000, os quais serviram de base para a construção de um indicador de vulnerabilidade social das famílias brasileiras, levando em consideração informações sobre o grau de inserção educacional, o grau de inserção económica local e o grau de inserção habitacional. Os resultados alcançados e as comparações efetuadas com o Índice de Desenvolvimento Humano (IDH) mostraram-se convincentes e validaram o indicador adotado pelos pesquisadores, o qual se revelou robusto e capaz de apontar diferenciais intermunicipais e intramunicipais no tocante à vulnerabilidade social. 0 estudo levou os autores a concluírem que o Índice de Vulnerabilidade Municipal (IVM) é sensível às condições de vida e ao desenvolvimento das localidades e regiões, o que the confere a característica de indicador sócioespacial. Representações cartográficas temáticas são utilizadas para ilustrar a vulnerabilidade social à escala nacional (municípios do Brasil) e municipal (bairros que compõem a Região Metropolitana de Belo Horizonte - MG).

Um exemplo de uso de geoprocessamento em estudos de vulnerabilidade social para a metrópole paulistana foi realizado por Alves (2006), no qual a vul- 
nerabilidade socioambiental foi estudada por meio de indicadores ambientais, na escala mais desagregada possível, ou seja, dos setores censitários. 0 autor detetou áreas críticas, nas quais há forte concentração de problemas e riscos sociais e ambientais com maiores níveis de população pobre e com privação social, tendo, portanto, menor capacidade de reação às situações de eventos perigosos, ou seja configurando-se como áreas de alta vulnerabilidade socioambiental.

O descrito nessa síntese bibliográfica aponta para a adequação do uso das geotecnologias em estudos de vulnerabilidade social e ambiental, os quais devem ser um dos pilares de políticas públicas focalizadas para as populações residentes em áreas de maior vulnerabilidade, seja à escala local, regional ou nacional ${ }^{1}$.

\section{Procedimento metodológico}

A metodologia adotada para a análise da vulnerabilidade dos 17 concelhos baseou-se em técnicas estatísticas de análise fatorial, que incluiu aspetos económicos, sociais e ambientais estudados, utilizando um programa de análise estatística para as Ciências Sociais, o SPSS R.18, associado ao Sistema de Informação Geográfica (SIG), programa ArcGIS 9.3. Essa metodologia baseia-se nos estudos de CutTer (1996, 2003); MENDES et al. (2009) e CunHA et al. (2011), conforme descrição anteriormente realizada. Nesse estudo a Vulnerabilidade Social resulta do produto da Criticidade com a Capacidade de Suporte, determinadas através de uma soma ponderada dos principais fatores, ponderação baseada na percentagem da variância explicada por cada um deles e com desagregação geográfica por concelho.

$\mathrm{Na}$ Análise Fatorial $^{2}$ executada no SPSS adotou-se como método de extração a Análise por Componentes

1 Tais experiências nortearam os procedimentos metodológicos adotados na pesquisa que serviu de base para a elaboração desse artigo, o Projeto de Pós-Doutoramento Geotecnologias aplicadas na Análise da Vulnerabilidade Social e Ambiental: um estudo metodológico comparativo entre Portugal e Brasil com financiamento do CNPq - Brasil. Este artigo possui interface com o Projeto de Pós-Doutoramento, financiado pela FCT, Cartografia geomorfológica de pormenor aplicada ao Ordenamento do Território - estudo na plataforma litoral e relevos adjacentes entre a Serra da Boa Viagem e a Nazaré, desenvolvido por Anabela Ramos, ambos supervisionados, no âmbito do CEGOT, por Lúcio Cunha.

${ }^{2}$ Apesar, de neste caso, termos um reduzido número de caso (apenas 17 municípios) e um elevado número de variáveis (45), o que é pouco adequado a uma análise fatorial, tendo em conta o comportamento das variáveis em termos de normalidade, linearidade e homocedasticidade, optámos por esta técnica de análise estatística, na falta de outra que possa aglutinar e sintetizar informação estatística sobre a sociedade e o ambiente dispersa por um conjunto tão variado de parâmetros. Por outro lado, pretendeu-se seguir as abordagens metodológicas referidas anteriormente, de modo a poder comparar resultados.
Principais, como método de rotação a rotina Varimax com Normalização Kaiser.

Tendo por base a análise de documentos cartográficos oriundos do Atlas do Ambiente Digital, produzido pela Agência Portuguesa do Ambiente ${ }^{3}$, assim como os levantamentos censitários do Instituto Nacional de Estatística $^{4}$, foi possível cartografar as principais variáveis indicadoras dos riscos socioambientais aos quais as populações de tais áreas estão expostas e espacializálas por meio de cartas temáticas elaboradas em ambiente de SIG com o programa ArcGIS.

Com vista a elucidar as características do objeto de estudo e os detalhes do desenvolvimento da metodologia adotada no trabalho, apresenta-se a caracterização da área de estudo, os procedimentos relacionados com a seleção das variáveis concernentes à Criticidade e Capacidade de Suporte, bem como as etapas para a realização da Análise Fatorial e a Classificação dos seus resultados em SIG visando elaborar a Carta de Vulnerabilidade Socioambiental.

\subsection{Caracterização da área de estudo}

Os 17 concelhos em estudo, da Região Centro de Portugal, compõem as sub-regiões NUT III do Baixo Mondego (na sua totalidade), Médio Tejo, Oeste, Pinhal Litoral (parcialmente), conforme exposto a seguir:

- Sub-região Baixo Mondego: Cantanhede, Coimbra, Condeixa-a-Nova, Figueira da Foz, Mira, Montemor-o-Velho, Penacova e Soure;

- Sub-região Médio Tejo: Alcanena e Vila Nova de Ourém;

- Sub-região Oeste: Alcobaça e Nazaré;

- Sub-região Pinhal Litoral: Batalha, Leiria, Marinha Grande, Pombal e Porto de Mós.

A localização da área de estudo é apresentada na Figura 1.

Para a seleção da referida área foram considerados os seguintes critérios:

- A sua localização numa faixa territorial contínua, com diferentes paisagens que vão desde a planície costeira até aos primeiros relevos interiores. Este aspecto visa salvaguardar o paralelo com o cenário brasileiro, que será alvo do estudo futuro, no âmbito do projeto de pesquisa que é base para o artigo;

${ }^{3}$ Agência vinculada ao Ministério da Agricultura, do Mar, do Am biente e do Ordenamento do Território http://sniamb.apambiente.pt/ webatlas/index.html. Acesso em 14/02/2012.

4 http://www.ine.pt/xportal/xmain?xpid=INE\&xpgid=ine_main. Acesso em 14/02/2012. 


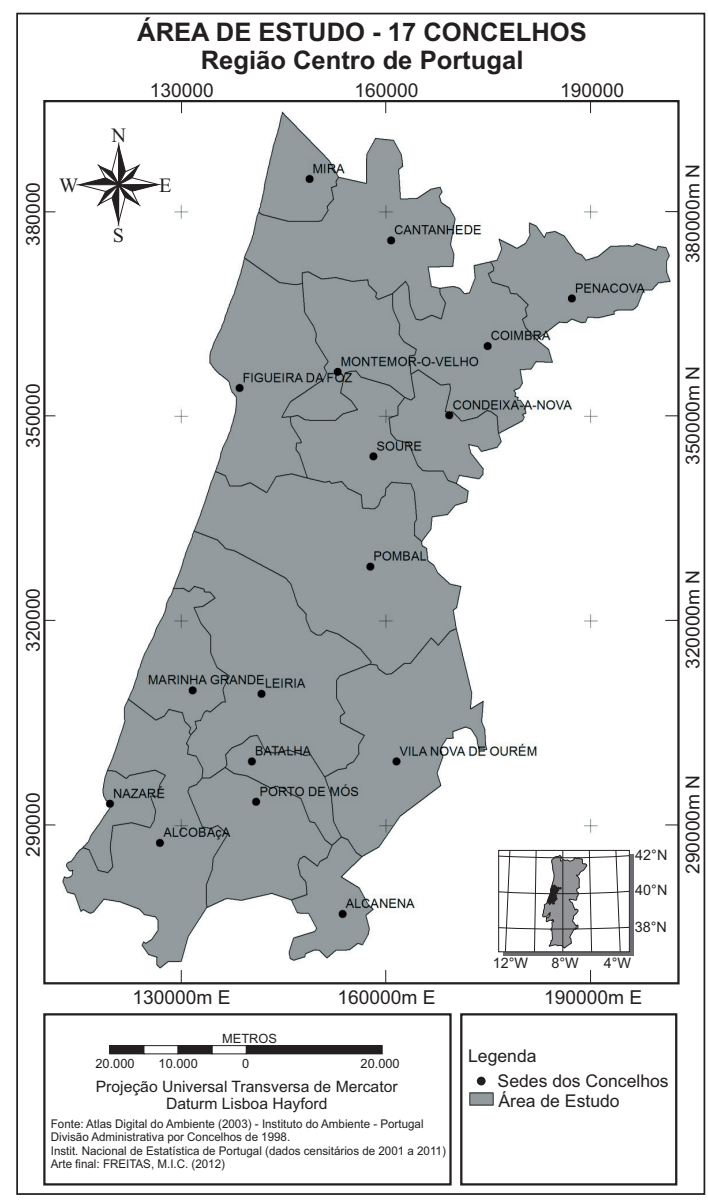

Figura 1

Área de estudo referente aos 17 concelhos da Região Centro de Portugal.

- Vizinhança física e situação social e económica diversificada;

- Ocupar sensivelmente a mesma área de um outro projeto em curso, com vista à caracterização da suscetibilidade e da perigosidade a riscos naturais (RAmos et al., 2011).

Os 17 concelhos abrigam um total de 731847 habitantes, e as populações concelhias variam de 13295 habitantes (Mira) a 131446 habitantes (Coimbra), de acordo com censo publicado pelo Instituto Nacional de Estatística (INE) de Portugal em 2011. Quanto aos aspetos socioeconómicos desses concelhos destacase Coimbra, cidade sede de distrito, com a base de sua estrutura urbana centrada na Universidade e em outras escolas e institutos de educação superior públicos e privados que permitem a frequência na cidade de cerca de 35000 estudantes. Tem na educação e na saúde os seus principais serviços, o que a torna uma das mais importantes cidades de Portugal. Além disso, o distrito de Coimbra detém 5.441 empresas, com uma facturação anual de 2.318 M€ (milhões de euros), embora apenas 83 delas estejam entre as mil maiores empresas do país, de acordo com os dados de 2005 (INE, 2011). O concelho de Leiria é o segundo mais populoso da área de estudo, com 129745 habitantes, possui como principal setor económico o setor terciário dos serviços e, também, fábricas de objetos de cerâmica e vidro. Leiria é a sede da NUT III do Pinhal Litoral, cujas atividades económicas são predominantemente urbanoindustriais, concentradas no comércio e serviços, com a maioria dos seus concelhos predominantemente agrícolas e rurais. Os dois concelhos (Coimbra e Leiria) concentram cerca de $36 \%$ da população total da área de estudo, valor indicativo não só de maior densidade populacional, mas também de maior dinamismo socioeconómico. Os demais concelhos em estudo tem um caráter predominantemente rural ou urbanorural, com atuação e características decorrentes de sua localização geográfica como é o caso, por exemplo, de Figueira da Foz, Nazaré e Marinha Grande, com atividades económicas voltadas para pesca, turismo, cultura da vinha e hortifruticultura.

Os concelhos de menor dimensão demográfica, com desenvolvimento menos expressivo, têm nos seus centros urbanos maior concentração de atividades do setor terciário, a par com pequenos parques industriais de pluriatividade, geralmente em complemento às atividades primárias vinculadas à pecuária e silvicultura, nas áreas de topografia mais acidentadas, e à agricultura do milho e do arroz, da vinha e hortifruticulturas nas planícies e vales.

Por meio da análise fatorial relativa à criticidade e capacidade de suporte pretende-se avaliar os resultados relativos da vulnerabilidade socioambiental da área estudada, com vista a observar o desempenho do modelo no contexto da realidade dessa região.

\subsection{Criticidade}

Para a análise da criticidade realizou-se um levantamento dos dados censitários respeitantes aos aspetos da vida da população, na busca de características dos concelhos em estudo que dessem indicadores de rutura do sistema, no caso de eventos perigosos associados a riscos tecnológicos ou naturais. Foram inicialmente selecionadas 108 variáveis, de acordo com os grupos indicados no Quadro I, onde se pode observar o número de variáveis explicativas para cada um dos grupos de variáveis selecionadas. 
Quadro I

Grupos de variáveis relativas à criticidade.

\begin{tabular}{|l|c|c|c|}
\hline \multirow{2}{*}{ Grupos } & \multicolumn{3}{c|}{ Variáveis } \\
\cline { 2 - 4 } & Iniciais & Modelo & Explicativas \\
\hline Economia e Condições de Vida & 38 & 16 & 9 \\
\hline População & 28 & 8 & 5 \\
\hline Saúde e Proteção Social & 14 & 9 & 2 \\
\hline $\begin{array}{l}\text { Condições de Alojamento } \\
\text { e dos Edifícios }\end{array}$ & 21 & 7 & 1 \\
\hline Educação & 5 & 3 & 2 \\
\hline Ambiente & 2 & 2 & 2 \\
\hline Total & 108 & 45 & 21 \\
\hline
\end{tabular}

Da execução do modelo de análise fatorial resultaram 21 variáveis explicativas das quais foi possível selecionar 5 fatores que detêm $76 \%$ da variância acumulada, considerando-se os 17 concelhos em estudo. As comunalidades das variáveis foram todas superiores a 0,88 .

De acordo com Martinez e Ferreira (2010) "as comunalidades estão relacionadas com a representação de uma variável para o fator. Numa outra perspectiva, representa a fidelidade do valor da variável. Um valor elevado na comunalidade indica que aquele item dá um grande contributo para a formação dos fatores extraídos" (ob. cit.: 153).

De posse de tais dados realizou-se a exportação para o programa ArcGIS dos resultados relativos aos 5 fatores mais significativos para cada concelho em estudo, realizando-se a sua espacialização tendo como referência os limites dos concelhos do Atlas do Ambiente Digital. A Criticidade foi calculada com ponderação baseada na percentagem da variância explicada pelos fatores de acordo com a seguinte expressão (1):

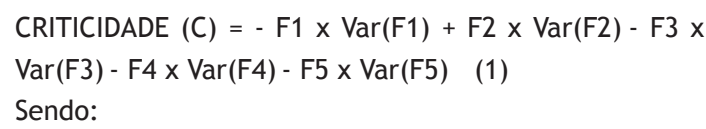

$\mathrm{FN}=$ Fatores resultantes da análise de Componentes Principais, $\mathrm{FN}=1 \ldots 5$.

$\operatorname{Var}(\mathrm{FN})=$ Percentagem da variância explicada pelos fatores, com $F N=1 \ldots 5$.

A variação dos sinais (positivo ou negativo) está diretamente associada ao significado das principais variáveis que definem cada fator, levando-se em conta que a maior Criticidade diz respeito a maior probabilidade de rutura do sistema e, consequentemente, de maior vulnerabilidade com respeito à variável ali explicitada.

Após o cálculo da Criticidade realizou-se a representação temática por meio da classificação em 5 grupos de seus resultados, aplicando-se numa primeira aproximação o classificador Natural Breaks (Jenks) seguido de classificação manual, para pequenos ajustes visando melhorar a distribuição dos concelhos por classe.

\subsection{Capacidade de suporte}

Em procedimento análogo, realizou-se o estudo da capacidade de suporte dos 17 concelhos em estudo, levantando-se os dados censitários que indicassem o nível de infraestruturas territoriais que permitem a reação das comunidades em caso de desastre associado a risco natural ou tecnológico.

Foram selecionadas 84 variáveis, de acordo com os grupos indicados no Quadro II, que apresenta o número de variáveis explicativas para cada um dos grupos de variáveis selecionadas, no caso da capacidade de suporte.

Quadro II

Grupos de variáveis relativas à capacidade de suporte.

\begin{tabular}{|c|c|c|c|}
\hline \multirow{2}{*}{ Grupos } & \multicolumn{3}{|c|}{ Variáveis } \\
\cline { 2 - 4 } & Iniciais & Modelo & Explicativas \\
\hline Condições dos Alojamentos e Edifícios & 32 & 18 & 9 \\
\hline $\begin{array}{c}\text { Infraestruturas para Monitorização } \\
\text { Territorial e Ambiental }\end{array}$ & 24 & 12 & 2 \\
\hline Condições Econômicas & 11 & 8 & 3 \\
\hline Condições de Vida da População & 11 & 3 & 1 \\
\hline Condições Agrícolas & 2 & 2 & - \\
\hline Infraestruturas de Saúde & 4 & 2 & 1 \\
\hline Total & 84 & 45 & 16 \\
\hline
\end{tabular}

Da execução do modelo de análise fatorial resultaram 16 variáveis explicativas das quais foi possível selecionar 5 fatores que detém 68\% da variância acumulada, considerando-se os 17 concelhos em estudo. As comunalidades das variáveis foram sempre superiores a 0,83 , o que aponta para o seu significativo contributo para a formação dos fatores.

A Capacidade de Suporte foi calculada com ponderação baseada na percentagem da variância explicada pelos fatores de acordo com a expressão (2):

CAPACIDADE DE SUPORTE (CS) = F1 $x \operatorname{Var}(F 1)+F 2 \times$ $\operatorname{Var}(F 2)+F 3 \times \operatorname{Var}(F 3)-F 4 \times \operatorname{Var}(F 4)+F 5 \times \operatorname{Var}(F 5)+F 6 \times$ $\operatorname{Var}(\mathrm{F6})$

Sendo:

$\mathrm{FN}=$ Fatores resultantes da análise de Componentes Principais, $\mathrm{FN}=1 \ldots 6$.

$\operatorname{Var}(\mathrm{FN})=$ Percentagem da variância explicada pelos fatores, com FN - 1...6.

De forma análoga ao exposto para a Criticidade, após o cálculo da Capacidade de Suporte realizou-se a 
sua representação temática por meio da classificação de seus resultados, aplicando-se numa primeira aproximação o classificador Natural Breaks (Jenks) seguido de classificação manual.

\subsection{Vulnerabilidade socio-ambiental}

$\mathrm{Na}$ posse dos dados relativos à Criticidade e à Capacidade de Suporte, realizou-se o cálculo da Vulnerabilidade Socioambiental dos 17 concelhos tenho em conta a expressão matemática (3):

$$
\mathrm{VS}=\mathrm{C} \times \mathrm{CS}
$$

Sendo:

VS = Vulnerabilidade Socioambiental

$\mathrm{C}=$ Criticidade

CS = Capacidade de Suporte.

De acordo com as classes definidas para a Criticidade e para a Capacidade de Suporte, realizou-se o produto entre esses dois mapas no programa ArcGIS, respeitando-se a definição das classes de vulnerabilidade socio-ambiental e legenda de tons de cinza apresentadas no Quadro III.

Com base nesses elementos foi possível a elaboração de mapas temáticos e gráficos ilustrativos da vulnerabilidade sócioambiental e de seus componentes, conforme descrição a ser apresentada no item resultados.

\section{Resultados}

\subsection{Criticidade}

No que concerne à Criticidade, o fator 1 , que explica $25 \%$ da variância, está relacionado com a economia e condições de vida da população, dando indicação dos concelhos com maior acesso ao crédito à habitação e maior taxa de emprego. Em acréscimo à situação económica está a baixa taxa de analfabetismo e a evasão da população das áreas rurais. Deve-se destacar que o

\section{Quadro III}

Classes de vulnerabilidade socio-ambiental (VS) e legenda de tons de cinza.

\begin{tabular}{|c|c|c|c|c|c|c|c|c|c|c|}
\hline \multirow{2}{*}{$\begin{array}{l}\text { Classes } \\
\text { de VS }\end{array}$} & \multirow{2}{*}{ Legenda } & \multirow{2}{*}{$\begin{array}{l}\text { Intervalo } \\
\text { Classe }\end{array}$} & \multirow{2}{*}{$\begin{array}{c}\text { Tons } \\
\text { de } \\
\text { Cinza }\end{array}$} & \multirow{3}{*}{ 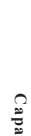 } & & \multicolumn{5}{|c|}{ Criticidade } \\
\hline & & & & & & 1 & 2 & 3 & 4 & 5 \\
\hline 1 & Muito Baixa & 1 a 3 & & & 1 & 1 & 2 & 3 & 4 & 5 \\
\hline 2 & Baixa & 4 a 6 & & 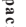 & 2 & 2 & 4 & 6 & 8 & 10 \\
\hline 3 & Média & 8 a 10 & & 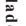 & 3 & 3 & 6 & 9 & 12 & 15 \\
\hline 4 & Alta & 12 a 16 & & E & 4 & 4 & 8 & 12 & 16 & 20 \\
\hline 5 & Muito Alta & 20 a 25 & & $\frac{7}{\sigma}$ & 5 & 5 & 10 & 15 & 20 & 25 \\
\hline
\end{tabular}

êxodo rural é uma situação recorrente não só na maioria dos concelhos em estudo, como em todo o País. Já o fator 2, que responde por $18 \%$ da variância total, diz respeito à população e à proteção social, dando destaque para as baixas condições de vida e baixa natalidade, expressas nos valores negativos das variáveis relativas aos alojamentos familiares com aquecimento central nas residências, à taxa de crescimento populacional efetivo e à população residente com 14 anos ou menos, um indício da baixa natalidade. Em síntese, observa-se que, enquanto o fator 1 enfatiza o aspecto económico, o fator 2 reflete questões relacionadas com a demografia dos concelhos. O Fator 3, que explica $13 \%$ da variância dos concelhos em estudo, trata da Economia, em termos das condições de comunicação, com destaque para os acessos telefónicos, ao baixo dinamismo quanto ao poder de compra e ao crédito à habitação. O Fator 4, que explica aproximadamente 10\% da variância, diz respeito às transformações no meio rural, ao enfatizar as explorações de áreas agrícolas com atividades não agrícolas, às receitas em ambiente dos municípios, que permite inferir sobre a diminuição do investimento em agricultura e a maior preocupação do poder público com as questões ambientais. O Fator 5, com 9\% de variância, trata da qualidade da Educação, expressa na baixa evasão escolar nos concelhos em estudo.

A Figura 2 apresenta os resultados da criticidade no gráfico de dispersão, relacionando o Fator $1 \mathrm{com}$ o Fator 2.

Da análise da Figura 2 pode-se observar que Coimbra, com muita vantagem com respeito a Leiria, Marinha Grande e Nazaré, destaca-se quanto à economia e Mira aparece como o concelho com menor dinamismo. 0 concelho de Montemor-o-Velho destaca-se quanto ao deficit na condição de vida e de presença da população infanto-juvenil enquanto Pombal apresenta melhores condições quanto a esses aspectos, ainda que

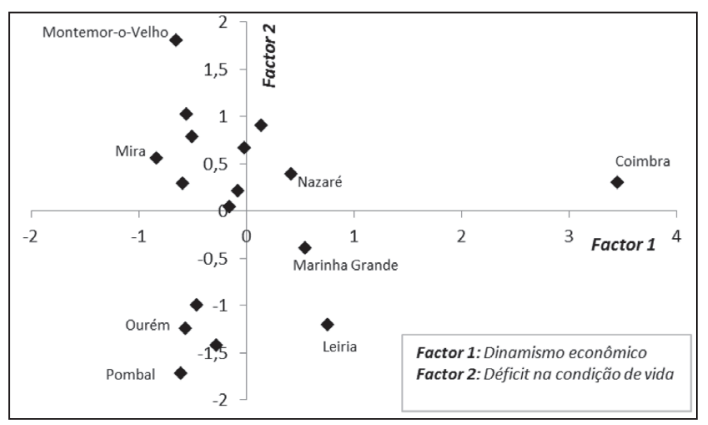

Figura 2

Criticidade relacionando Fator 1 com Fator 2. 
apresente baixo dinamismo económico, de acordo com as análises realizadas nessa pesquisa.

A Figura 3 ilustra a representação espacial da criticidade para os 17 concelhos da Região Centro de Portugal.

A análise da Figura 3 indica valores elevados a muito elevados de Criticidade para os concelhos do Norte e Noroeste da área de estudo, que apresentam menor dinamismo económico e nas condições de vida, principalmente nas taxas de natalidade e no êxodo rural, com destaque para Penacova, Soure e Montemoro-Velho. As exceções recaem sobre Coimbra e Condeixa-a-Nova que apresentam valores muito baixos. Já os valores baixos ou muito baixos de Criticidade predominam nos concelhos do Centro e Sul da região de estudo, com maior dinamismo económico e população menos envelhecida, com destaque para Alcobaça. A exceção fica em Alcanena, a Sudoeste da área de estudo, que apresenta Criticidade muito elevada.

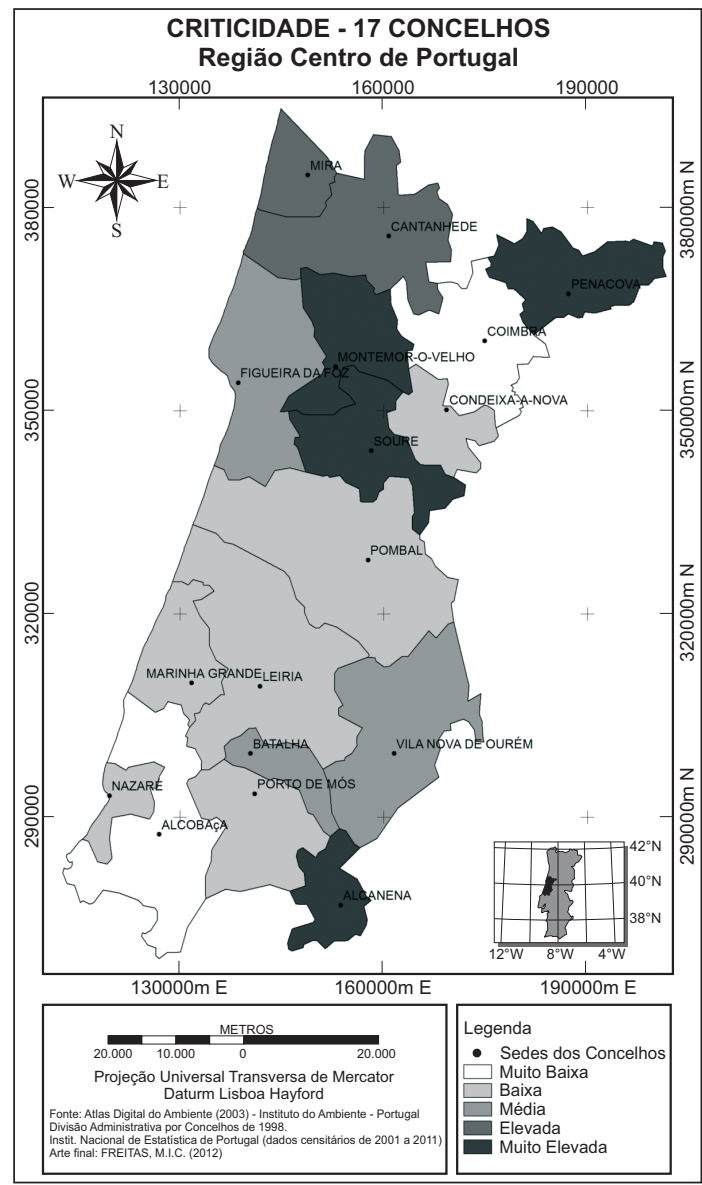

Figura 3

Criticidade para os 17 Concelhos da Região Centro de Portugal

\subsection{Capacidade de Suporte}

Quanto à Capacidade de Suporte observa-se que as principais variáveis explicativas do Fator 1, que concentra $20 \%$ da variância, estão relacionadas com as Condições Económicas, dando indicação dos concelhos com maior desenvolvimento de acordo com o número de empresas e estabelecimentos bancários. No que se refere aos aspetos das Infraestruturas de Alojamento representa a diminuição de famílias ocupando alojamentos individuais, indicando tendência para residências em condomínios de edifícios com multiplas habitações. Além disso, aponta para a carência de Infraestruturas para Monitorização Territorial e Ambiental no que concerne à presença de Bombeiros. O Fator 2, que responde por $14 \%$ da variância total, diz respeito às Condições dos Alojamentos, destacando a presença de alojamentos com aquecimento central. As variáveis seguintes tratam, respetivamente, de edifícios com necessidade de reparação e alojamentos superlotados e são ambas negativas, indicando as boas condições das infraestruturas habitacionais. Em síntese, observa-se que o fator 1 enfatiza a capacidade de suporte em termos de condições económicas enquanto o fator 2 destaca as condições de alojamento e qualidade de vida da população.

o Fator 3, que explica 13\% da variância dos concelhos em estudo, trata do Envelhecimento dos Edifícios e da Presença de Alojamentos Vagos, permitindo inferências sobre o abandono de imóveis envelhecidos nos concelhos que se destacam quanto a esse aspecto. O Fator 4, que explica aproximadamente $12 \%$ da variância, diz respeito à baixa proporção de edifícios novos, indicativo da desaceleração na construção civil, enquanto o Fator 5, que explica $8 \%$ da variância, aponta a presença de edifícios de uso sazonal.

A Figura 4 apresenta os resultados da capacidade de suporte no gráfico de dispersão relacionando fator 1 com o fator 2 para os concelhos em estudo.

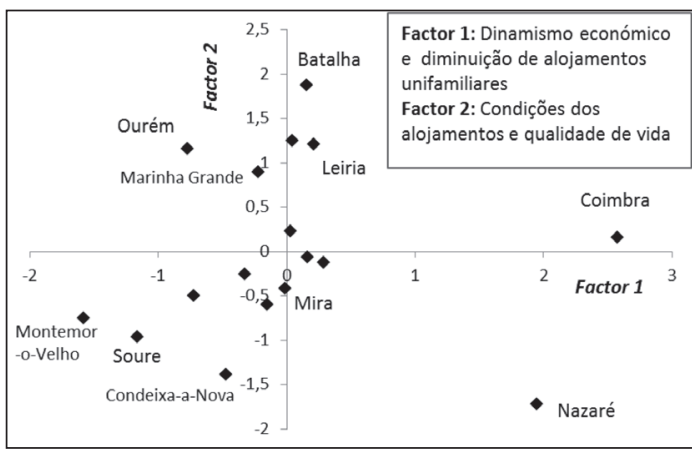

Figura 4

Capacidade de suporte relacionando Fator 1 com Fator 2 . 


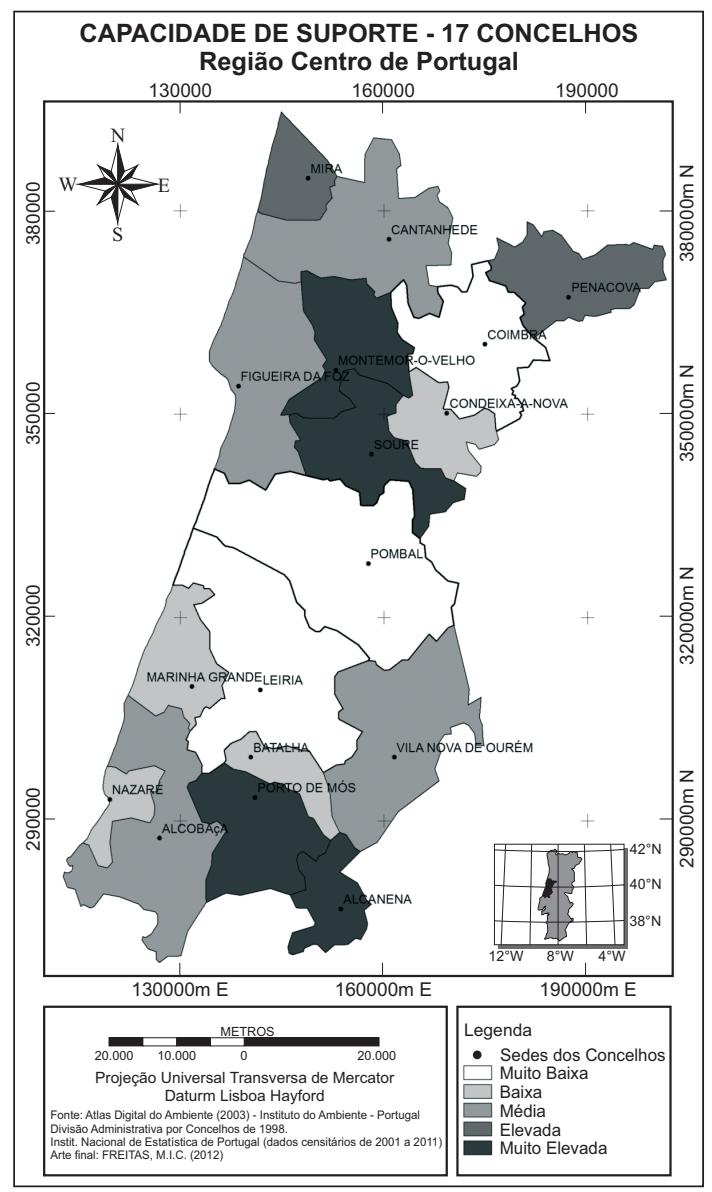

Figura 5

Capacidade de suporte dos 17 concelhos da Região Centro de Portugal

Da análise da Figura 4 pode-se observar que Coimbra, seguida de Nazaré, Leiria e Batalha destacam-se quanto ao dinamismo económico e diminuição de alojamentos unifamiliares, com Montemor-o-Velho em última posição. Quanto às Condições dos Alojamentos e Qualidade de Vida destacam-se os concelhos da Batalha, Ourém, Leiria, Marinha Grande, com Nazaré em última posição.

A Figura 5 ilustra a representação espacial da Capacidade de Suporte.

\subsection{Vulnerabilidade socioambiental}

A Figura 6 apresenta a representação espacial dos resultados obtidos no que se refere à Vulnerabilidade Socioambiental dos 17 concelhos em estudo.

Os resultados obtidos com este estudo indicam como principais fatores de Vulnerabilidade Socioambiental as condições relativas ao baixo dinamismo eco-

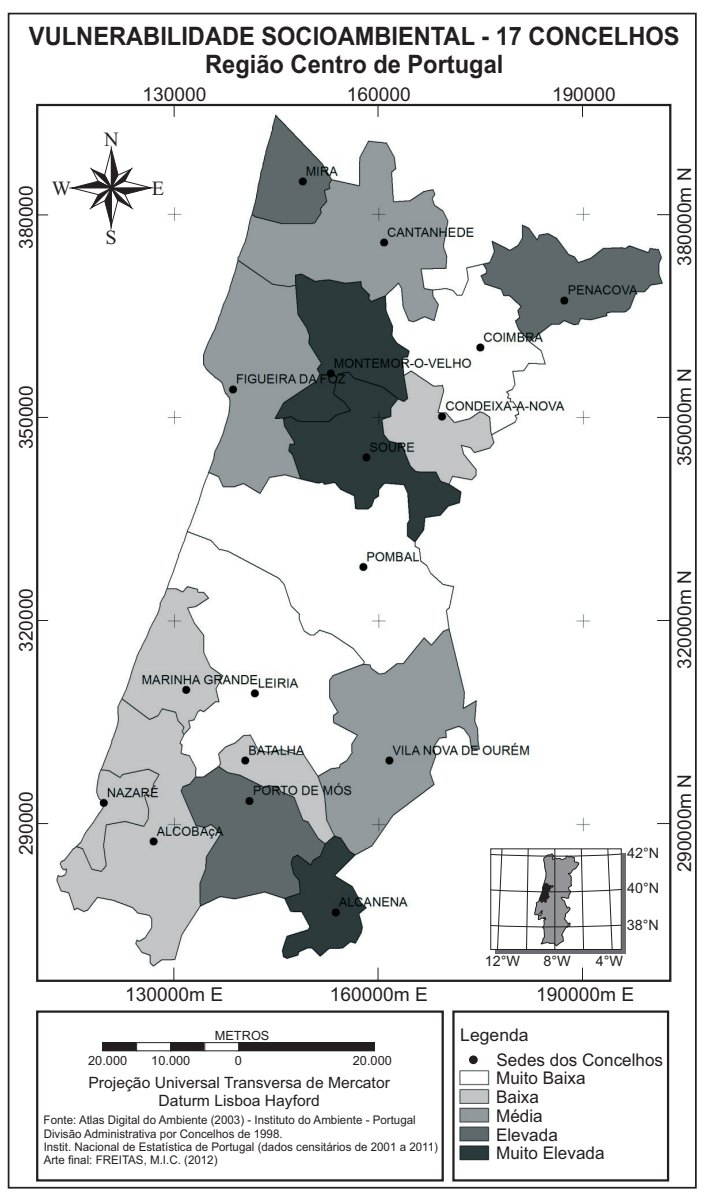

Figura 6

Vulnerabilidade Socioambiental dos 17 concelhos da Região Centro de Portugal

nómico e aos déficits demográficos, principalmente relacionados com a população infantojuvenil e com o êxodo rural. Este é um indicador do envelhecimento da população que é questão recorrente nos municípios de Portugal, o que se confirma para a área de estudo. Os valores altos ou muito altos de Vulnerabilidade aparecem para concelhos do setor Norte (Montemor-o-Velho, Soure, Mira e Penacova), com menor desenvolvimento económico e condições ambientais e infraestruturais menos desenvolvidas para enfrentar situações de risco. Também com esse perfil estão os concelhos do setor Sul da área de estudo como Alcanena e Porto de Mós.

Já os concelhos com baixa Vulnerabilidade Socioambiental são aqueles que apresentaram melhor Capacidade de Suporte em termos de economia concentrada nos ambientes urbanos, bem como aqueles que concentram maior número de indivíduos em alojamentos multifamiliares. Os concelhos de Coimbra, Pombal e Leiria apresentam muito baixa Vulnerabilidade Socioambien- 
tal enquanto valores de vulnerabilidade baixa são apresentados para concelhos do Centro e Sul da área de estudo: Marinha Grande, Alcobaça, Nazaré e Batalha.

\section{Considerações finais}

Como considerações finais deste artigo destacase a metodologia adotada, que se apresenta acessível e facilmente replicável no contexto de Portugal.

A análise privilegia alguns aspectos socioeconómicos e ambientais em detrimento de outros, devido à limitação nos dados censitários disponíveis, o que se observou no número expressivo de variáveis que tiveram de ser eliminadas do modelo devido aos dados incompletos para a maior parte dos concelhos em estudo. A falta de informação de muitas variáveis, principalmente as relativas aos aspectos de qualidade de vida e ambiente, é um problema que precisa ser enfrentado por parte do poder público. Da mesma forma, os pesquisadores que desenvolvem trabalhos na temática devem estar atentos para resultados que possam ser superestimados ou subestimados devido a essas ausências.

O processo de modelação fazendo uso da Análise Fatorial e a sua representação cartográfica através de cartas temáticas permite uma visão de síntese dos concelhos e constitui uma importante base para o planeamento de ações de prevenção, enfrentamento de riscos e Vulnerabilidades Socioambientais. Espera-se que os resultados alcançados com esse estudo possam servir de base para futuras análises de detalhe que possibilitem a elaboração de propostas de políticas públicas relacionadas com as vulnerabilidades aqui cartografadas.

\section{Agradecimentos}

Os autores agradecem à Doutora Zélia Barroso Fernandes e ao Doutor Rui Gama Fernandes o prestimoso auxílio no tratamento, através de análise factorial, da informação estatística.

\section{Referências}

Alves, H. P. F. A. (2006) - "Vulnerabilidade socio-ambiental na metrópole paulistana: uma análise sociodemográfica das situações de sobreposição espacial de problemas e riscos sociais e ambientais". Revista Brasileira de Estudos da População, São Paulo, v. 23, n. 1. pp. 43-59.

Beier, C. e Dowing, T. E. - Geografía y ayuda humanitária. Bilbao, Universidad de Deusto. Instituto de Derechos Humanos. 1998.

Blaikie, P.; Cannon, T.; Davis, I. e Wisner, B. (1994) - At risk. Natural hazards, people's vulnerability and disasters. Routledge, London. 284p.

Cunha, L.; Mendes, J. M.; Tavares, A. e Freiria, S. (2011) "Construção de modelos de avaliação de vulnerabilidade social a riscos naturais e tecnológicos. 0 desafio das escalas". In: Santos, N. e Cunha, L. (org.) - Trunfos de uma Geografia Activa. Imprensa da Universidade de Coimbra, Coimbra. pp. 627-637.

CUtTeR, S. L. (1996) - "Vulnerability to environmental hazards". Progress in Human Geography, vol. 20, $n^{\circ}$ 4. Thousand Oaks, CA. pp. 529-539.

CUTTER, S. L. et al. (2003) - "Social vulnerability to environmental hazards". Social Science Quarterly, vol. 84, $\mathrm{n}^{\circ} 2$. Oklahoma. pp. 242-261.

Garcia, R. A. e Matos, R. (2007) - "A distribuição espacial da vulnerabilidade social das famílias brasileiras". In: Anais do Seminário População, Pobreza e Desigualdade. Belo Horizonte: UFMG. pp. 1-20.

Instituto Nacional de Estatísticas - INE. (2011) - Censo 2011. http:// censos.ine.pt $/$ xportal $/ x$ main? $x p i d=C E N S O S \& x p g i d=c e n s$ os2011_apresentacao (acesso em 14/02/2012).

Martinez, L. F. e Ferreira, A. I. (2010) - Análise de Dados com SPSS: Primeiros Passos. Escolar Editora, Lisboa. 177 p.

Mendes, J. M.; Tavares, A.; Cunha, L. e Freiria, S. (2009) "Vulnerabilidade social aos riscos naturais e tecnológicos em Portugal". In: Guedes Soares, C.; Jacinto, C.; Teixeira, A. P. e ANTÃo, P. (org.) - Riscos Industriais e Emergentes. Edições Salamandra, Lisboa. pp. 67-84.

Ramos, A. M.; Cunha, L. S. e Cunha, P. P. (2011) - "Diferenciação territorial e caracterização dos riscos naturais na área entre a Figueira da Foz e a Nazaré". Atas do VIII Congresso da Geografia Portuguesa, Lisboa, APG Edts. CD-Rom. 6 p.

United Nations - International Strategy for Disaster Reduction UNISDR. (2009) - Global Plataform 2011. 2009. http://www. unisdr.org/. Acesso em 10 de agosto de 2010.

United Nations - International Strategy for Disaster Reduction UNISDR. (2011) - Disaster Risk Reduction in United Nations: Roles, mandates and areas of work of key UN entities. Ed. UNISDR, Geneva. 155 p. http://www.unisdr.org/ files/18933_directory.pdf (acesso em 2 de fevereiro de 2012). 